\title{
Double stages of feature extarction-based GFPMI for colored finger vein identification
}

\author{
Dawlat Mustafa Sulaiman ${ }^{1}$, Adnan Mohsin Abdulazeez ${ }^{2}$, Habibollah Haron ${ }^{3}$ \\ 1,2Department of Information Technology, College of Informatics, Duhok Polytechnic University, Iraq \\ ${ }^{3}$ Department of Computer Science Faculty of Computing, University Teknologi Malaysia, Malaysia
}

\begin{tabular}{l} 
Article Info \\
\hline Article history: \\
Received Jul 15, 2019 \\
Revised Oct 18, 2019 \\
Accepted Nov 8, 2019 \\
\hline
\end{tabular}

Keywords:

Classification

Finger vein

Glcm features

Gray scale texture features

Identification

Localization

Residual neural network

\begin{abstract}
Today, finger vein recognition has a lot of attention as a promising approach of biometric identification framework and still does not meet the challenges of the researchers on this filed. To solve this problem, we propose s double stage of feature extraction schemes based localized finger fine image detection. We propose Globalized Features Pattern Map Indication (GFPMI) to extract the globalized finger vein line features basede on using two generated vein image datasets: original gray level color, globalized finger vein line feature, original localized gray level image, and the colored localized finger vein images. Then, two kinds of features (gray scale and texture features) are extracted, which tell the structure information of the whole finger vein pattern in the whole dataset. The recurrent based residual neural network $(\mathrm{RNN})$ is used to identify the finger vein images. The experimental show that the localized colored finger vein images based globalized feature extraction has achieved the higher accuracy (93.49\%) while the original image dataset achieved less accuracy by (69.86\%).
\end{abstract}

Copyright $(0) 2020$ Institute of Advanced Engineering and Science. All rights reserved.

\section{Corresponding Author:}

Dawlat Mustafa Sulaiman,

Department of Information Technology,

University of Duhok Polytechnic, Duhok, Iraq.

Email: dawlat.mustafa@dpu.edu.krd

\section{INTRODUCTION}

The term 'biometrics' refers strictly to a science involving the statistical analysis of biological characteristics. Here, biometrics is used in a context of analyzing human characteristics for security purposes. This measurable characteristic, biometric, can be physical, such as eye, face, finger image, hand or behavioral, like signature and typing rhythm [1-2]. It is often said that with biometric products you are able to reach the highest level of security. To help illustrate this point, a definition is used by the biometrics industry [3]. Basically, finger vein image has an identical pattern in which the blood vessels are constructed as a unique network under the skin of the human finger. Each individual that has a unique pattern even though the twin has different vein vessels pattern [4-5]. In contrast, the hand vessels and palm have also applied in the human biometric identification filed but they still do not have a significant performance to be more popular in this filed [6].

Some techniques such as hand recognition are used for performing accurate recognition [7-8]. Some significant advantages such as higher resistance to identify the criminal, higher performance (identification accuracy), significant less time consuming and higher speed of authentication. The complexity of the finger vein feature extraction still a big challenge for the researcher to produce such a strong system that able to distinguish between different finger vein pattern from the same person among different ones. In this paper, a higher finger vein identification/verification system based double stages of localized and globalized feature extraction of grayscale and colored finger vein is proposed using Globalized Features Pattern Map Indication (GFPMI). The propose system relies on double feature extraction stages based on propose a Globalized 
Finger Vein Features Pattern Map Indication (GFPMI) scheme to detect and extract the localized finger vein feature lines based on using the localized finger vein images that has been detected and extracted using our first approach [9]. Then, use those lines in the second stage to extract set of significant features which are used later for the classification approach to distinguish between different pattern of the finger vein that belong to the same person.

Different techniques has been proposed by many different researches to solve the finger vein identification and verification based on using different approaches such as globalized feature extraction based supervised learning using K- nearest neighbor algorithm (KNN) classification algorithm, or localized feature descriptor based convolutional neural network (CNN). Convolutional neural network is an instance of deep learning strategy is mimicking brain function in processing in-formation [9]. Ruqaiya at el. [10] proposed and develop globalization approach for finger vein recognition system-based K- nearest neighbor algorithm (KNN) classification approach and finger vein image analysis tools. They rely on the KNN to verify and calculate the performance of the recognition system after some globalized features are extracted from the finger vein images. Experimentally, the proposed system for finger vein verification system based KNN achieved accuracy starting from 55.84\% till 92.21\%. Yu at el. [11] proposed a convolutional neural network $(\mathrm{CNN})$ based local feature descriptor for finger vein recognition. The proposed system showed that the convolutional neural network based local feature descriptor ( $\mathrm{CNN}-\mathrm{CO})$ can exploit and extract the discriminative finger fine features. Basically, the complex $\mathrm{CNN}$ filter (kernels) are strong enough to find the corresponding local features base Gabor filter banks. The experimental results of the proposed system show that finger vein recognition system based local feature descriptor demonstrated effectiveness performance results when it has been applied to two public finger vein datasets. Manmohan at el. [12] proposed a finger vein recognition system based maximum edge position detection. The proposed system is based on the feature collection stage that use the sign and the magnitude of the finger vein edges which illustrate and capitalize the differences of the gradient feature values in different directions. The proposed maximum values of the finger vein edges based different gradient directions show that the classification accuracy has achieved $89.47 \%$ and approximately error rate up to $12.48 \%$. Kashif Shaheed at. el. [13] proposed a finger vein recognition system based of behavioral and physiological attributes. It is based on analyzing finger vein pattern image based on some novel techniques that have been proposed before for feature extraction. The proposed system based on difrenet stages such as finger vein image acquisition, pre-processing, feature extraction and diferent matching methods. The experimental results showed that accurate results based on the comparative studies for finger vein image recognition. Manisha Sapkale at. el. [14] proposed an embedded finger vein recognition and classification system for human authentication. The proposed system based on a novel finger vein image recognition system. The proposed malgoithm is based on using the Gabor filtering for finger vein feature extraction and based on the distance neajoirty for finger vein classification. The expermentail results showed that finger vein recognition become more reliable, easier and accurate.

\section{METHDOLOGY}

The finger vein image identification and verification system that is propose in this paper has three main stages. The first stage is based mainly on using unsupervised learning-based on optimized k-means clustering that has been proposed in our first model [9]. The finger vein images are localized and detected as well as the binary mask is been generated which will be used later for the feature extraction stage. The second stage of the proposed system is the localized finger vein feature extraction. In this stage, the finger vein lines are extraction based on propose an approach we called Globalized Finger Vein Pattern Map Indication (GFVPMI). The proposed approach based on the localized finger vein images to extract the indicate the vein lines in the finger vein image. After that, we project the extracted features on the main finger vein images and generate different type of finger vine image datasets such as the original finger vein images, localized finger vein images, and colored finger vein images. A summary of the propose system is illustrated in Figure 1.

GFVPMI basis first on construct the localized image filtering that is used to filter the whole finger vein image globally. Conceptually, it relies of the localized frequency ridge lines of each finger vein image (local vein lines). The idea of the finger vein frequency ridge lines is build based on the idea of extracting the vein line orientation based on the local image filtering. The orientation of each finger vein is defined as the valley between two finger vein lines which in this case we will used the finger vein gradient image based on using Sopel Operator. This step allows us to estimate and approximate the magnitude of the gradient image after converting the whole localized finger vein image (ROI based on the localized binary mask) to continuous vector and extract the localized ridge orientation. The main framework of our propose algorithm Globalized Finger Vein Pattern Map Indication (GFVPMI) is illustrated in Algorithm 1. 


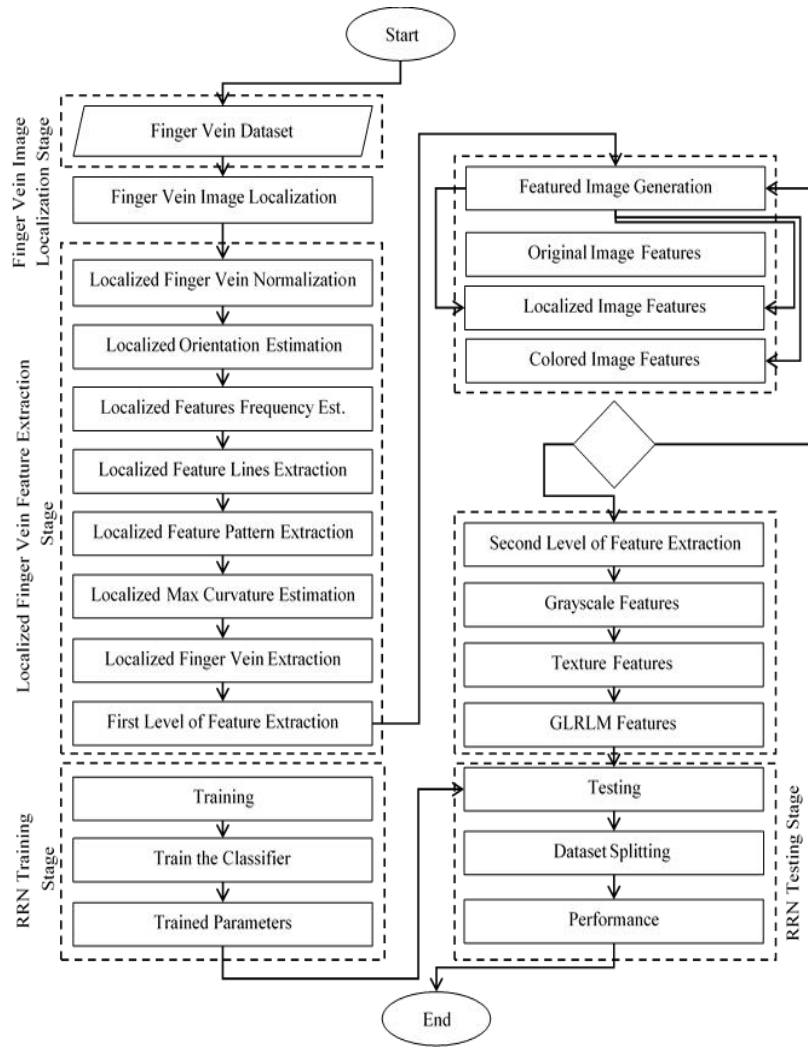

(a)

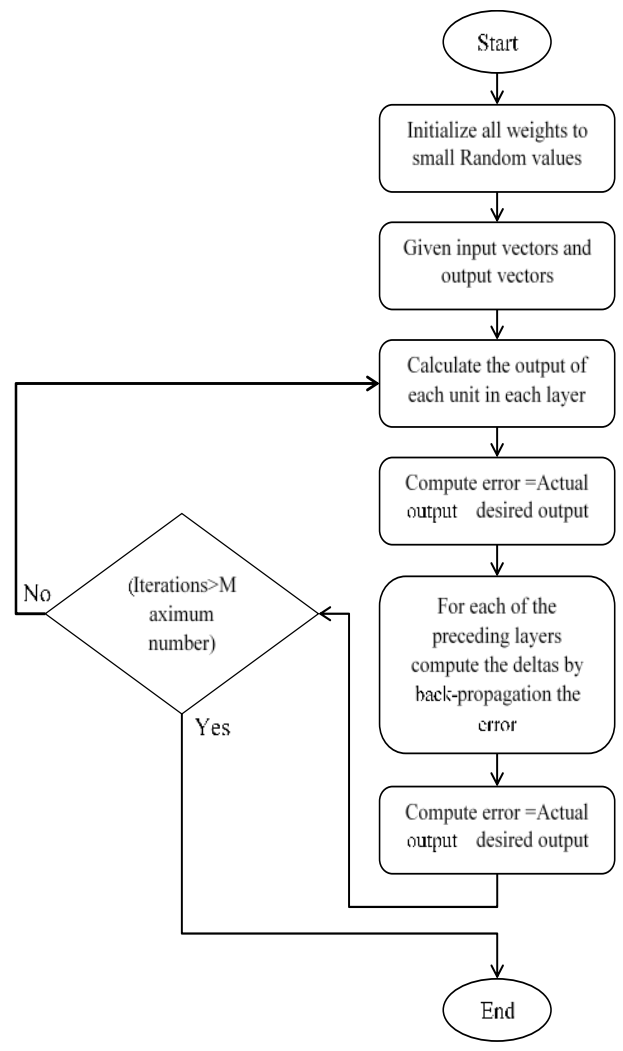

(b)

Figure 1. (a) Double Stages of Feature Extarction-Based Localized and Globalized Features Pattern Map Indication Based Colored Finger Vein Identification System Flowchart: The dashed lines illustrate each individual stage such as the Finger Vein Image Localizatiion, the Localized Finger vein Feature Extraction, and Localized Finger Vein Image Recognition and Classification, (b) The Recurrent Residual Neural Network (RNN) Block diagram: Dashed lines illustares the recursive function for fine tune the parameter during the training phase

\begin{tabular}{ll}
\hline Algorithm 1 Globalized Finger Vein Pattern Map Indication (GFVPMI) \\
\hline Inputs: Localized Finger Vein image \\
Output: First level of globalized Features \\
\hline 1. $\begin{array}{l}\text { Step 1: Do the localized moralization based on estimated (pre-specified) } \\
\text { mean and variance are computed to normalize the finger vein images. }\end{array}$ \\
2. $\begin{array}{l}\text { Step 2: Estimated the localized finger vein line orientation based on using } \\
\text { the normalized finger vein images. }\end{array}$ \\
3. Step 3: Extract the localized finger Vvein lines Based on using globalized \\
map.
\end{tabular}

To extract the GFVPMI that is used mainly for localized finger vein feature extraction map, some steps are implemented and used such as.

\subsection{Localized Image Normalization}

In this step, we used the localized image normalization that basis on estimated the local mean $M$ and local variance $\operatorname{Var}$ based on using the intensity area of the finger vein ROI that has been detected using our first model [9]. The main formal of localized image normalization of the finger vein images is shown in (1) [15].

$$
\mathcal{N}(i, j)=\sqrt{\frac{\operatorname{Var}_{l}\left(\mathcal{J}(i, j)-M_{l}\right)^{2}}{\operatorname{Var}}}
$$

Where $M_{1}$ and $\operatorname{Var}_{l}$ iare the localized mean and variance of the finger vein image based on using the determine ROI area to compute them. 


\subsection{Estimate the Localized Features Orientation}

In term of estimated the localized feature orientations, the coordinates of the local feature lines neighborhoods of the globalized map that is extracted in the GFVPMI is used. Basically, each finger vein image is normalized using the localized image normalization step, then the image gradient is computed based on using the Sopel Operator. Let assume that the $I(i, j)$ is the original localized finger vein image, and $N(i, j)$ is the normalized image. Then, $G(i, j)$ is the sopel operatos image gradien as the (2) shows below [15].

$$
|G(i, j)|=\sqrt{G_{x}(i, j)^{2}+G_{y}(i, j)^{2}}
$$

where $|G(i, j)|$ is the absolute of the gradient image. The gradient image is donated as $G x(i, j)$ and $G y(i, j)$ which $G x(i, j)$ illustrates and detect the all the pixels in the x-axis and $G y(i, j)$ illustrates and detect all the pixels in the y-axis. Then, the gradient image magnitude is approximated based on using (3).

$$
|G|=\left|G_{x}(i, j)\right|+\left|G_{y}(i, j)\right|
$$

Moreover, the localized finger vein feature orientations are estimated based on using local block that is assumed to be centered in the center of each valley which is the intersection between each finger vein lines and the Least Square Estimation (LSE) uing (4), (5) and (6) [16].

$$
\begin{aligned}
& \mathcal{V}_{x}(i, j)=\sum_{u=i-\frac{w}{2}}^{i+\frac{w}{2}} \sum_{i-\frac{w}{2}}^{j+\frac{w}{2}} 2 G_{x}(u, v) 2 G_{y}(u, v) \\
& \mathcal{V}_{y}(i, j)=\sum_{u=i-\frac{w}{2}}^{i+\frac{w}{2}} \sum_{i-\frac{w}{2}}^{j+\frac{w}{2}}\left(G_{x}^{2}(u, v)-G_{y}^{2}(u, v)\right) \\
& \theta(j, j)=\frac{1}{2} \tan ^{-1}\left(\frac{v_{y}(i, j)}{v_{x}(i, j)}\right)
\end{aligned}
$$

Where the $\theta(j, j)$ is denoted as the Least Square Estimation (LSE) of the orientation for each local ridge in each block. Finally, the $2 \mathrm{D}$ finger vein image is converted to a continuous vector filed, by using (7) and (8).

$$
\begin{aligned}
& \Phi_{x}(i, j)=\cos (2 \theta(i, j)) \\
& \Phi_{y}(i, j)=\sin (2 \theta(i, j))
\end{aligned}
$$

Then, the localized finger vein lines orientation are extracted using (9).

$$
\mathcal{O}(i, j)=\frac{1}{2} \tan \left(\frac{\Phi_{x}^{\prime}(i, j)}{\Phi_{y}^{\prime}(i, j)}\right)
$$

The main steps of the local based feature orientation estimation algorithm are shown in Algorithm 2.

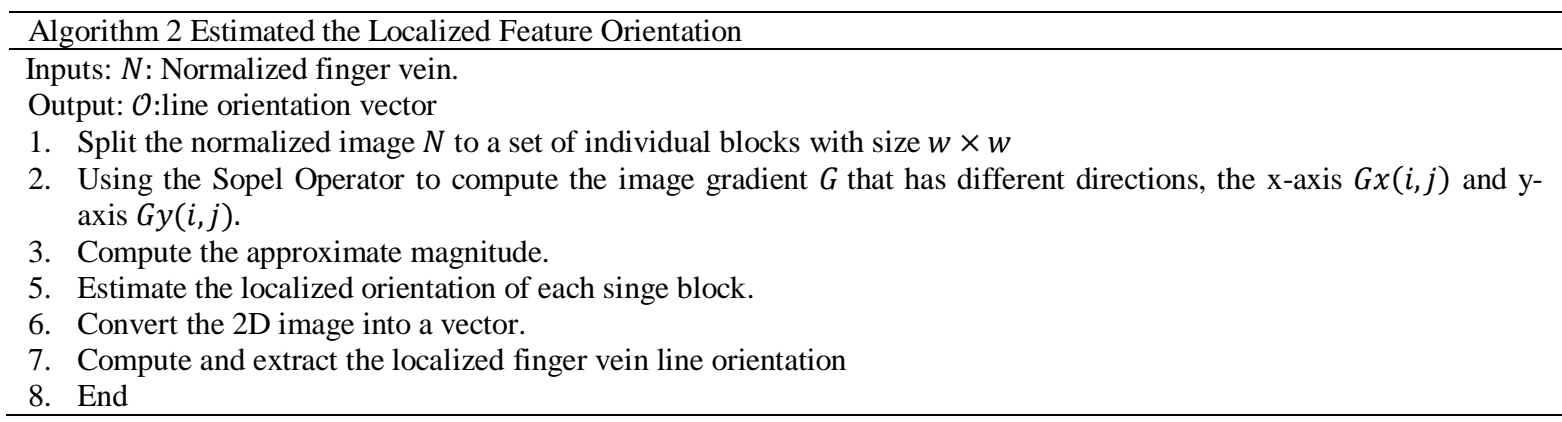

The results of the estimated the localized feature orientation step are shown in Figure 2. Figure 2(a) shows the original finger vein image, Figure 2(b) shows the Globalized Finger Vein Pattern Map Indication GMFPI map. And Figure 2(c) shows the localized finger vein image after project the Globalized Finger Vein Pattern Map Indication GMFPI map on the orgibal localized finger vein image. 


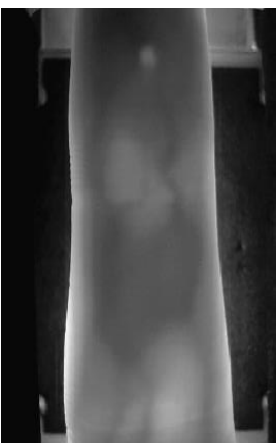

(a)

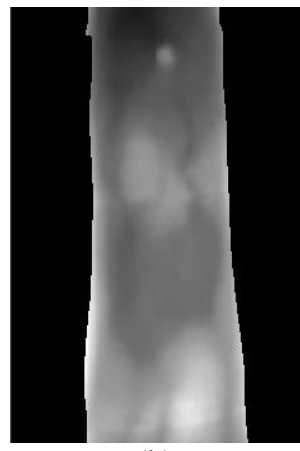

(b)

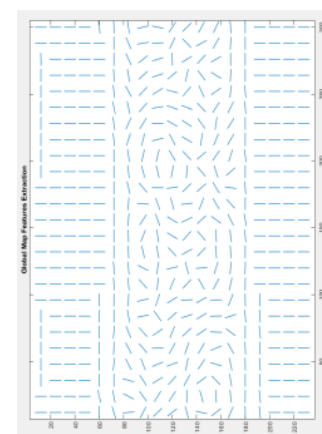

(c)

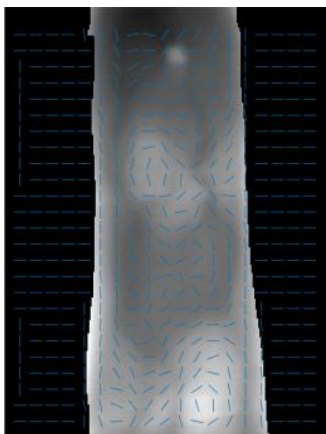

(d)

Figure 2. Estimated the Localized Feature Orientation. (a) original Finger Vein Image, (b) Localized finger vein image, (b) the Globalized Finger Vein Pattern Map Indication GMFPI map. (c) Localized Finger vein image based Globalized Finger Vein Pattern Map Indication GMFPI map projection

\subsection{Globalized Finger Vein Lines Extraction Based (GMFPI):}

Finger vein feature extraction model based the globalized feature orientation map is based on determine starting preprocessing image which is basically relies on the starting point by lower half of the input finger vein image using (10) [15-16].

$$
\operatorname{mask}=\operatorname{zeros}\left(\operatorname{mask}_{h}, \operatorname{mask}_{w}\right)
$$

Where mask $_{h}$, mask $_{w}$ denoted as the lower half of the original input finger vein image size. Then, the preprocessing filtering mask is constructed based on using (11) and (12).

$$
\begin{aligned}
& \text { mask }=\sum_{i=1}^{h / 2} \text { mask }=-1 \\
& \text { mask }=\sum_{i=\frac{h}{2}}^{n} \text { mask }=1
\end{aligned}
$$

The filter kernels based on the selected sigma value by setting the mask size $x$ and $y$ based on using (13).

$$
\text { kernel }=\frac{1}{2 \times \pi \times \sigma} e^{-\frac{x^{2}+y^{2}}{2 \times \sigma^{2}}}
$$

Then, the original finger vein image is processed based on (14).

$$
\text { Image }_{\text {filtered }}=\operatorname{Conv}(\text { image, } \text {,ernel })
$$

The rest steps of the globalized finger vein lines extraction are described in Algorithm 3.

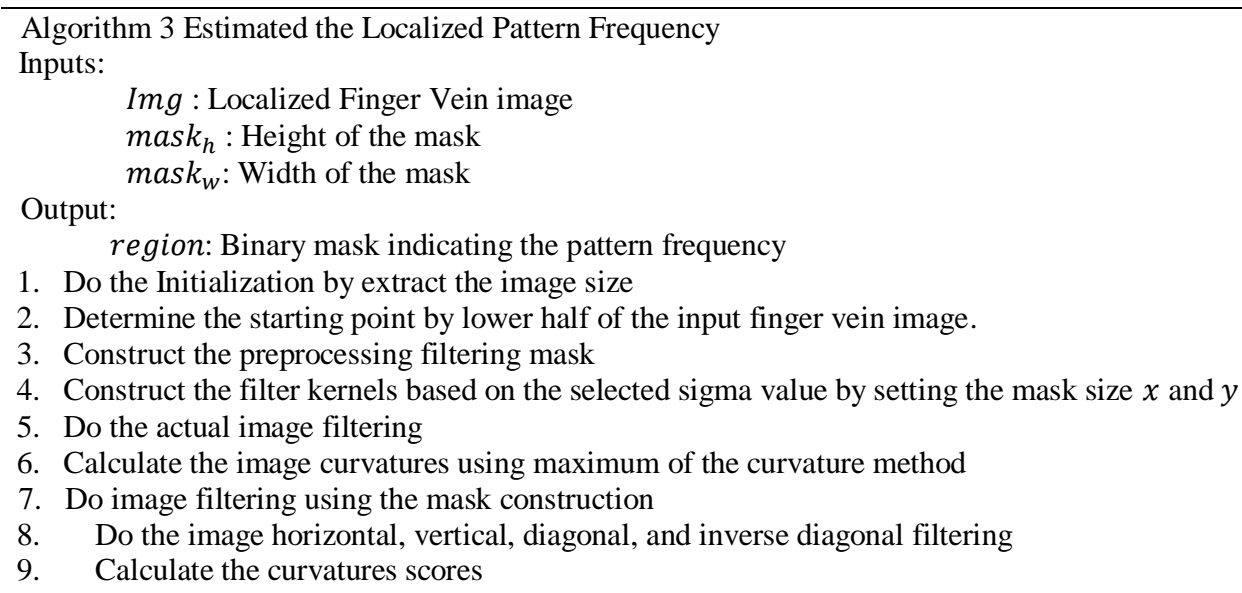


10. Extract the horizontal, vertical, and diagonal direction

11. Extract the finger vein image lines using repeated line detection and tracking

12. Set the probability of the left or right

13. Set the probability of going up or down

14. Calculate the locus space

15. Check if the width is even

16. Extract the $r$ for oblique directions

17. Extract the half width for horizontal. and vertical directions

18. Extract the half width for oblique directions

19. Omit the unreachable borders

20. Uniformly distributed the starting points

21. For $i=1$ to $n$ do $/ *$ Iterate through all starting points $* /$

22. Determine the moving-direction attributes

23. Determine the moving candidate point

24. Detect the dark line direction near current tracking point

25. Register the tracking information

26. Increase the value of tracking space

27. Move the tracking point

28. End for

29. Extract and binarize the vein lines image (binary mask) using mean average

30. Binarize the vein image using (mean average)

31. Binarize the vein image using (median)

32. Do the image closing using line str with 0 degree

33. Remove the small object pixels from the binary image

34. End

The result of the first step of the finger vein feature (lines) extraction (image filtering using kernel mask construction) is shown in Figure 3(a), (b), (c) and (d). Figure 3(a) illustrates the horzeontal finger vein feature lines direction, while Figure 3(b) shows the vertical finger vein feature direction. Figure 3(c) and (d) illustrate the digional and updiognal of finger vein feature direction respectively.

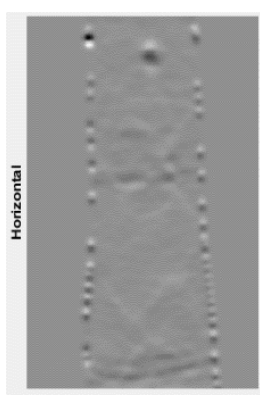

(a)

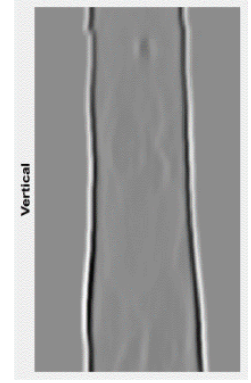

(b)

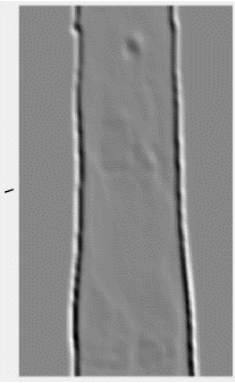

(c)

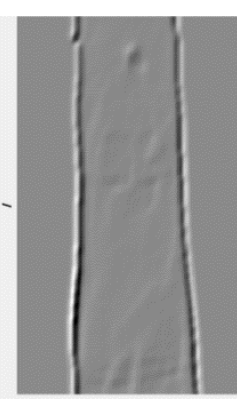

(d)

Figure 3. Localized finger vein feature (lines) extraction-based finger vein image filtering using kernel mask construction). (a) Horzeontal finger vein feature lines direction, (b) Vertical finger vein feature direction, (c) and (d) are the digional and updiognal of finger vein feature direction respectively

Although, the result of the second step of the finger vein feature extraction based on extract the curvatures lines is shown in Figure 4. Figure 4(a) shows the binarized result of the horzeontal finger vein binarized feature lines direction, Figure 4(b) shows the binarized results of the vertical finger vein binarized feature direction. Figure 4(c) and (d) show the binarized results of the digional and updiognal of finger vein feature direction respectivelly. The binary mask generation of the vein lines in the finger vein image using (mean and median) is shown in Figure 4(e) and (f) respectively. The finger vein image prop veins lines using repeated line detection and tracking is shown in Figure 4(g). Then, binarize the vein lines image (binary mask) step result is shown in Figure 4(h). Finally, the binary mask of the finger vein image is shown in Figure 4(e) where some small objects are removed the image cleaning using image closing is applied. We used to generate different finger vein image datasets such as the original finger vein dataset, the localized finger vein dataset, the localized feature extracted finger vein images datasets, and finally the colored localized feature extracted dataset. are shown in Figure 5. 
Figure 5(a) shows the the original finger vein image, Figure 5(b) shows the localized finger vein image, Figure 5(c) shows the globalized finger vein feature based gray scale finger vein image, and Figure 5(d) shows the globalized finger vein feature based colored localized finger vein image.

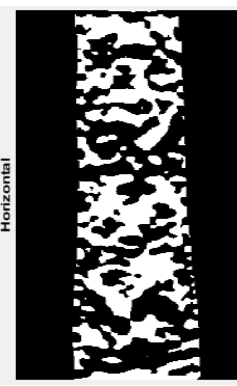

(a)

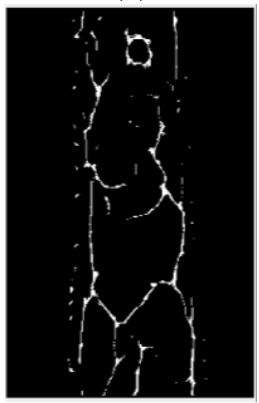

(e)

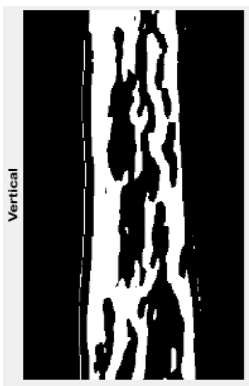

(b)

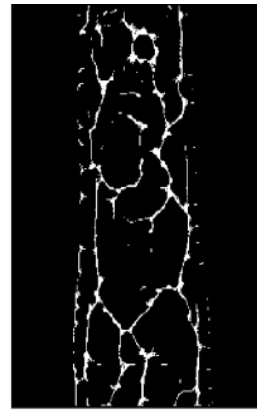

(f)

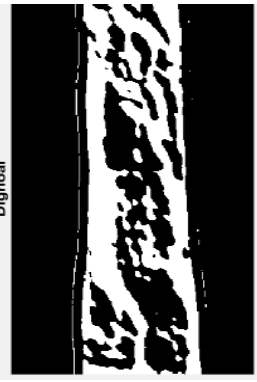

(c)

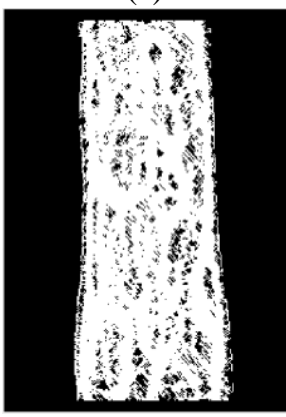

(g)

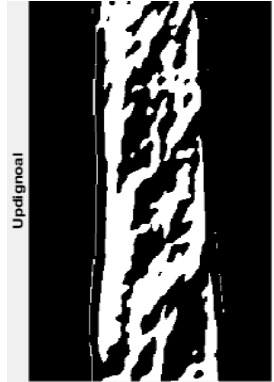

(d)

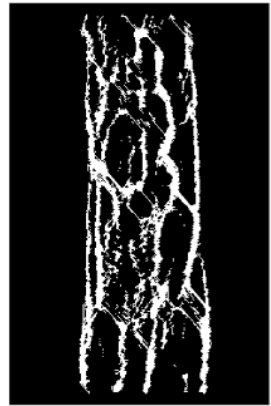

(h)

Figure 4. Localized binarization of the finger vein feature (lines) extraction-based finger vein image filtering using kernel mask construction). (a) Binarized horzeontal finger vein feature lines direction, (b) Binarized vertical finger vein feature direction, (c) and (d) are the Binarized digional and updiognal of finger vein feature direction respectively, Binarized mask generation of the vein lines in the finger vein image using mean (e)-(f) and median (g)-(h)

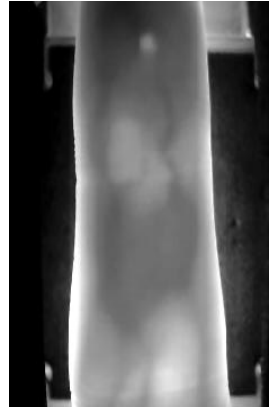

(a)

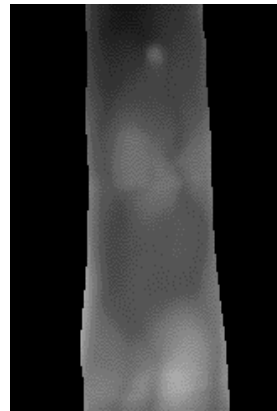

(b)

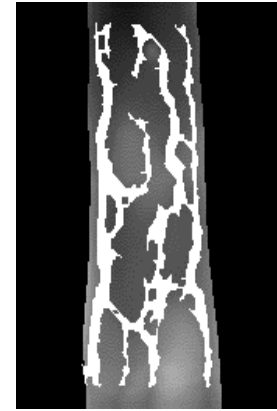

(c)

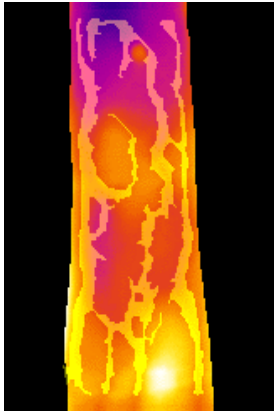

(d)

Figure 5. Different finger vein image datasets (a) the original finger vein dataset, (b) Localized finger vein image dataset, (c) Globalized finger vein feature based gray scale localized finger vein image, (d) Globalized finger vein feature based colored localized finger vein image

The second level of the feature extraction stage is basically based on the three type os features: structural featurtes, grayscale, and semetrical (texture) featurtes [17-18]. The second level of the extracted features extract some significant information about the that represents the raw image to facilitate decision making such as significant pattern for classification.

\subsubsection{Gray Scale Features}

The first grpoup of feature set that represent the structural featurtes. The set of the grayscale features are illustrated in 4 types of features such as mean, standard deviation (variance), kurtosis and skewness (15), (16), (17), and (19) [17]. 
Mean $=\sum x P(x)$

Standard Deviation $=\frac{\sqrt{\left(\sum(x-\bar{x})^{2}\right)}}{n}$

Kurtosis $=\left(\frac{1}{\text { variance }}\right) \sum_{x=1}^{m} \sum_{y=1}^{n}(f(x, y)-\text { mean })^{4}$

Skewness $=\left(\frac{1}{\text { variance }}\right) \sum_{x=1}^{m} \sum_{y=1}^{n}(f(x, y)-\text { mean })^{3}$

\subsubsection{Texture Features}

The second group of features is the textural features which is illustrated in 5 diferent features that are extracted from the co-occurrence matrices [17]. This second group of the grayscales feature's types are computed based on the statistical properties that are drived from the GLCM features matrix. The five textural features that are used in this group are Entropy, Correlation, Contrast, Energy, and Homogeneity that are illustrated in (19), (20), (21), (22) and (23) [19].

$$
\begin{aligned}
& \text { Entropy }=-\sum_{i=1}^{n} \sum_{j=1}^{m} p(i, j) \log (p(i, j) \\
& \text { Corr }=\frac{1}{(n-1)} \sum^{\left(x-\mu_{x}\right)\left(y-\mu_{y}\right) p(x, y)} \\
& \sigma_{x} \sigma_{y} \\
& \text { Contrast }=\sum_{x, y}|x-y|^{2} p(x, y)^{2} \\
& \text { Energy }=\sum_{x, y} p(x, y)^{2} \\
& \text { Homogeneity }=\sum_{x, y} \frac{p(x, y)}{1+|x-y|}
\end{aligned}
$$

\subsubsection{GLRLM Features}

The third group of the second feature level is the grayscale features. Here the GLRLM feature extraction technique is used. GLRLM is used to extract 11 features depending on the derive the gray level run length matrix (GLRLM) for two level high frequency sub bands of the discrete wavelet to decomposed image. First feature is 1 for distance. The second one is the degree which are 0,45,90 and 135 [17]. After that we compute the mean and variance for each feature to extend the total number of features to 27 features.

\subsection{Finger Vein Identifcation and Classification Stage}

Recurrent based Residual Neural Network (RNN) is the main classification algorithm that is proposed in our proposed system. The residual neural network (RNN) model is based on compos the state of the transition function $f$ to an output function $g$. Usually, RNN model is build and implemented using multilayer neural network. The standard RNN function is directly related to the positional a cyclic-based graphs which is a super-source node transition theory [21-22]. The standard RNN function is directly related to the positional a cyclic-based graphs which is a super-source node transition theory. The main structure of the RNN is the same of the graph structure as is shown in Figure 1(b). The basis transition function of the RNN is implemented based on the recursive state representation as is shown in (24) and (25) [21]:

$$
\begin{aligned}
& a(v)=f\left(a(\operatorname{ch}[v]), I(v), v, w_{f}\right) \\
& y(v)=g\left(a(v), v, w_{g}\right)
\end{aligned}
$$

Were $w_{f}$ and $w_{g}$ represent the model parameters (synaptic weights) of the network $f$ and $g$ respectively. RNN process the weight transition based on the graph $U$ in terms to adjust the synaptic weights. In this case, the whole network (graph) leads to encode the network in both learning and recall phase, for this reason it called a Residual Neural Network [23]. Basically, the feed-forward of the RNN is carried out the encoding part of the network where each node $v$ represent the transition function $f$, and $a(v)$ represents the state transition of the stat $v$. More specifically, the state $a(v)$ is computed by the transition function of the specific input label $I(v)$. The Pseudo-code of the RNN training based stochastic gradient descent is described in algorithm 4 [23]. 


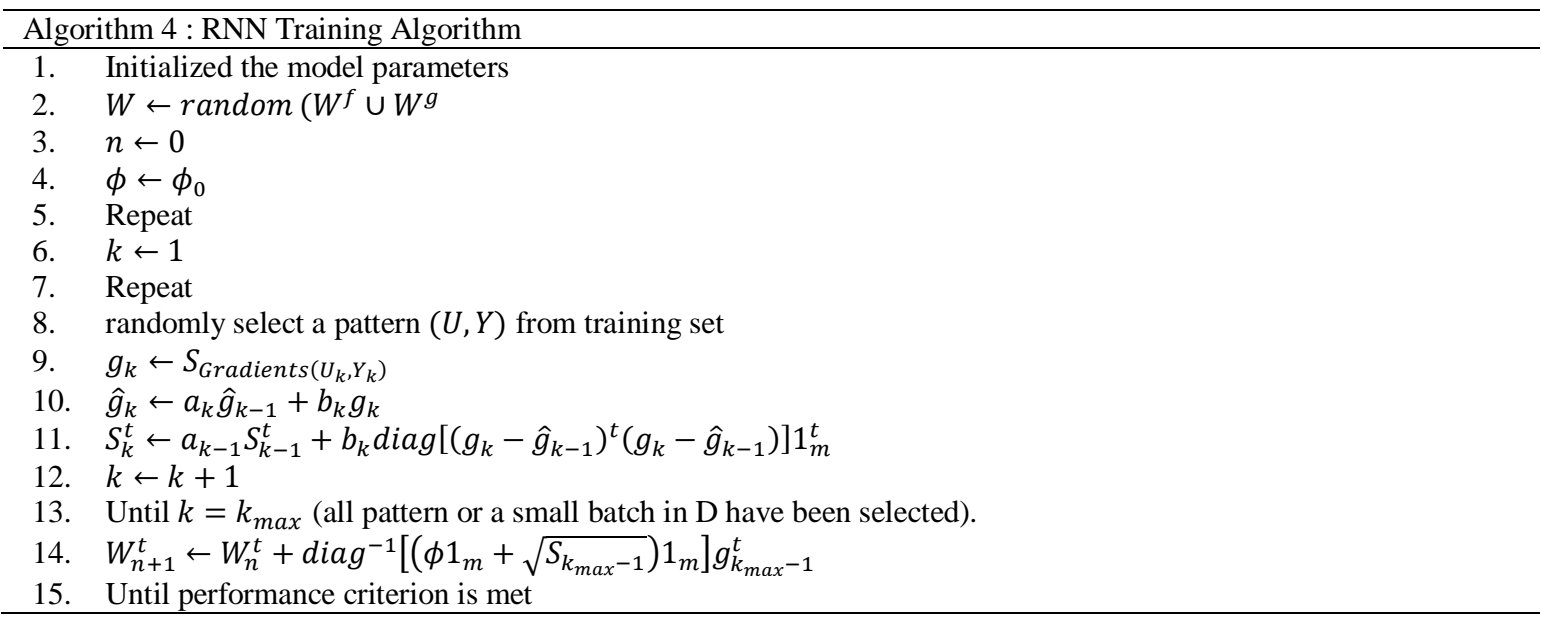

Conceptually, in the classification part, the input of the training formula consists of examples in the shape of feature vectors with a label appointed to them. The target of classification algorithm is to learn to assign correct labels to new unseen samples of constant task. A classification formula consists of two parts: a learning model and a classification module. The learning module builds a model supported a tagged training set. This model consists of designed by the training module and contains a group of associative mappings (e.g. rules). These mappings, once applied to associate untagged check instance, predict labels of the check set.

\section{EXPERMENTAL RESULTS}

\subsection{Dataset}

In this paper, we used the SDUMLA-HMT a finger vein database. This dataset has been captured based on the device that has been designed by Joint Lab for Intelligent Computing and Intelligent Systems of Wuhan University. The dataset has different cases, each case provides images of his/her index finger, middle finger and ring finger of both hands. The collection data for each of the 6 fingers is repeated for 6 times to obtain 6 finger vein images [24, 26-27].

\subsection{Training and Testing Set Selection}

We use 5-fold cross-validation, the best way to select the folds is by down sampling. We set our test size to a maximum of 20 percent of the dataset. This near equality allows for a more accurate evaluation of the resulting classifiers.

\subsection{Cross Validation Execution}

The training set was then divided into five folds; each fold equally having randomly selected images. One-fold is withheld for the validation step. The same already split folds are used to train and validate our classifiers and the performance of the trained classifier is decided by the votes collected from the classifier of each fold. The training, cross validation, and testing datasets are shown in Table 1.

Table 1. Total Number of Subject and Finger Vein Images in the Training and Testing Dataset

\begin{tabular}{|c|c|c|c|c|}
\hline \multirow[b]{2}{*}{ Subjects } & \multirow[b]{2}{*}{ Total } & \multicolumn{2}{|c|}{ Training $(80 \%)$} & \multirow[b]{2}{*}{ Testing (20\%) } \\
\hline & & Training $(60 \%)$ & Validation $(20 \%)$ & \\
\hline 10 & 360 & 216 & 72 & 72 \\
\hline
\end{tabular}

\subsection{Evaluation Criteria and Performance Results}

The evaluating performance of the finger vein identification and classification system is calculated by using three measures called Recognition Rate (RR), Precision (PR), Sensitivity (SE), Specificity (SP) [25, 28-29]. The performance results of the propose system (Finger vein identification based double feature extraction stages) are shown in Table 2. We notice that the localized colored finger vein image identification and recognition has achieved the higher accuracy in both training and testing by achieving (91.07\%) in the training part and $(94.49 \%)$ in the testing part. In contrast, the localized gray finger vein images have achieved less by reaching $(88.95 \%)$ in the training and $(89.86 \%)$ in the testing. Moreover, the localized finger fine 
image without the first globalized feature extraction stage achieved $(70.59 \%)$ in the training and $(76.16 \%)$ in the testing while the original datasets achieved $(69.25 \% 0$ in the training and $(69.86 \%)$ in the testing.

Table 2. Our Propose Performance Results

\begin{tabular}{cccccc}
\hline \multirow{2}{*}{ Image Model } & Training Accuracy & \multicolumn{4}{c}{ Testing } \\
\cline { 3 - 5 } & & Precession & Recall & F1-measue & Accuracy \\
Original & 69.25 & 73.00 & 71.93 & 67.50 & 69.86 \\
Localized & 70.59 & 75.10 & 77.06 & 75.09 & 76.16 \\
Localized_-gray & 88.95 & 86.67 & 87.14 & 86.30 & 89.86 \\
Localized-Colored & 91.07 & 88.95 & 89.36 & 88.75 & 94.49 \\
\hline
\end{tabular}

\section{CONCLUSION}

The propose system for finger vein image identification and recognition "Double stages of feature extraction based localized and globalized features pattern map indication (GFPMI) for colored finger vein image identification" shows significant performance when it applied on one of the most challenges finger vein dataset. The experimental results of the propose system show that our approach localized colored finger vein images based globalized feature extraction has achieved the higher accuracy (93.49\%) on the testing dataset comparting with the gray level localized finger vein image dataset that achieved less accuracy by $(89.86 \%)$. Our system shows that the experimental results of the finger vein identification can be more accurate if we based on the localized version of the finger vein image using our first approach [9]. We notice that the accuracy of the finger vein image identification based on using the localized finger vein images has achieved (76.16) without the globalized finger vein lines extraction (the first stage of our feature extraction system) while using the same system based on the original image dataset achieved less accuracy by $(69.86 \%)$. Performance and the accuracy of the designed system can accurately identify different pattern of finger vein for the same person among different subject on the whole dataset.

\section{REFERENCES}

[1] Sravya., V, Radha, K., Ravindra, B., and Srujana, B, "A Survey on Fingerprint Biometric System", International Journal of Advanced Research in Computer Science and Software Engineering, Vol. 2, Issue 4, April 2012.

[2] Haar, Helen, Darelle van Greunen, and Dalenca Pottas, "The characteristics of a biometric", IEEE In Information Security for South Africa, 2013, pp. 1-8.

[3] Malik, Iram, and Rohini Sharma, "Analysis of different techniques for finger-vein feature extraction", Int. J. Comput. Trends Technol (IJCTT) 5: 4, 2013.

[4] Kono, Miyuki, Naoto Miura, Takao Fujii, Koichiro Ohmura, Hajime Yoshifuji, Naoichiro Yukawa, Yoshitaka Imura et al. "Personal Authentication Analysis Using Finger-Vein Patterns in Patients with Connective Tissue Diseases-Possible Association with Vascular Disease and Seasonal Change." PloS one 10, no. 12 (2015): e0144952.

[5] Yang, Jinfeng, and Yihua Shi. "Towards finger-vein image restoration and enhancement for finger-vein recognition." Information Sciences, 268 (2014): 33-52.

[6] Bonneau, Joseph, and Sören Preibusch. "The Password Thicket: Technical and Market Failures in Human Authentication on the Web." WEIS. 2010.

[7] Mayyadah R. Mahmood, Adnan M. Abdulazeez, "A Comparative Study of a New Hand Recognition Model Based on Line of Features and Other Techniques", International Conference of Reliable Information, 2017.

[8] Mayyadah Ramiz Mahmood, Adnan Mohsin Abdulazeez, "Different Model for Hand Gesture Recognition with a Novel Line Feature Extraction", 2019 International Conference on Advanced Science and Engineering (ICOASE).

[9] Dawlat Mustafa Sulaiman, Adnan Mohsin Abdulazeez, Habibollah Haron, Shereen S Sadiq, "Unsupervised Learning Approach-Based New Optimization K-Means Clustering for Finger Vein Image Localization”, 2019 International Conference on Advanced Science and Engineering (ICOASE).

[10] R. Khanam, R. Khan and R. Ranjan, "Analysis of Finger Vein Feature Extraction and Recognition using DA and KNN Methods," 2019 Amity International Conference on Artificial Intelligence (AICAI), Dubai, United Arab Emirates,2019,pp.477-483.doi: 10.1109/AICAI.2019.8701253

[11] Y. Lu, S. Xie and S. Wu, "Exploring Competitive Features Using Deep Convolutional Neural Network for Finger VeinRecognition,"in IEEEAccess, vol.7, pp.35113-35123, 2019. doi: 10.1109/ACCESS.2019.2902429.

[12] Manmohan and V. Sharma, "Finger Vein Recognition Using Modified Maximum Edge Position Octal Patterns," 2019 6th International Conference on Signal Processing and Integrated Networks (SPIN), Noida, India, 2019,pp.287-292.doi: 10.1109/SPIN.2019.8711573.

[13] Kashif Shaheed 1 ID , Hangang Liu 2 , Gongping Yang 1,*, Imran Qureshi 1 , Jie Gou 1 and Yilong Yin, "A Systematic Review of Finger Vein Recognition Techniques", Information 2018, 9, 213; doi:10.3390/info9090213.

[14] Manisha Sapkale, S. M. Rajbhoj, "A finger vein recognition system", 2016 Conference on Advances in Signal Processing (CASP), Pune, India, 9-11 June 2016, 10.1109/CASP.2016.7746185.

[15] Wikipedia.n.d.Normalization (image processing). https://en.wikipedia.org/wiki/Normalization_(image_processing). 
[16] Bob HowisonJune,"Bob's Imaging Fundamentals \#3: Pseudocolor and LUTs”, 18, 2015, https://possibility.teledyneimaging.com/bobs-imaging-fundamentals-3-pseudocolor-and-luts.

[17] Shweta Jain, "Brain Cancer Classification Using GLCM Based Feature Extraction in Artificial Neural Network", IJCSET, ISSN: 2229-3345, VoL 4 No. 07 JuI2013.

$\begin{array}{llll}\text { [18] MooK. Chung, "GaussianKernelSmoothing", } & \text { July30, }\end{array}$ http://pages.stat.wisc.edu/ mchung/teaching/768/reading/lecture02-smoothing.pdf.

[19] Anja Attig, Petra Perner," A Comparison between Haralick's Texture Descriptor and the Texture Descriptor Based on Random Sets for Biological Images", Machine Learning and Data Mining in Pattern Recognition. Volume 6871 of the series Lecture Notes in Computer Science pp 524-538.

[20] Leyton, M. "Symmetry, Causality, Mind“. MIT Press, Massachusetts (1992).

[21] Churchland, P., Sejnowski, T. "The Computational Brain", MIT Press (1992).

[22] https://leonardoaraujosantos.gitbooks.io/artificialinteligence/content/residual_net.html.

[23] Yilong Yin, Lili Liu, and Xiwei Sun. n.d. "SDUMLA-HMT: A Multimodal Biometric Database." School of Computer Science and Technology, Shandong University.

[24] D. Al-Jumeily, A. Al-Azzawi, A. Mahdi and J. Hind. 2017. "A Robust Spatially Invariant Model for Latent Fingerprint Authentication Approach." 2017 10th International Conference on Developments in eSystems Engineering (DeSE). Paris.

[25] Adil Al-Azzawi, Hasanain Al-Sadr, Jianlin Cheng, Tony X Han,” Localized Deep Norm-CNN Structure for Face Verification", 2018 17th IEEE International Conference on Machine Learning and Applications (ICMLA).

[26] Adil Al-Azzawi, Jade Hind, Jianlin Cheng," Localized Deep-CNN Structure for Face Recognition", 2018 11th International Conference on Developments in eSystems Engineering (DeSE).

[27] Zeebaree, D. Q., Haron, H., \& Abdulazeez, A. M. (2018, October). "Gene Selection and Classification of Microarray Data Using Convolutional Neural Network". In 2018 International Conference on Advanced Science and Engineering (ICOASE) (pp. 145-150). IEEE.

[28] Zeebaree, D. Q., Haron, H., Abdulazeez, A. M., \& Zebari, D. A. (2019, April). "Trainable Model Based on New Uniform LBP Feature to Identify the Risk of the Breast Cancer". In 2019 International Conference on Advanced Science and Engineering (ICOASE) (pp. 106-111). IEEE.

[29] Borra, S. R., Reddy, G. J., \& Reddy, E. S. (2018). “An Efficient Fingerprint Identification using Neural Network and BAT Algorithm“. International Journal of Electrical \& Computer Engineering (2088-8708), 8(2). 